\title{
Aligned magnetic field and Navier slip effects on free convective radiative flow of nanofluids with imbedded carbon nanotubes: a Lie group analysis
}

\author{
P. Sreenivasulu ${ }^{1} \cdot$ S. R. Gunakala ${ }^{2} \cdot$ T. Poornima $^{3} \cdot$ N. Bhaskar Reddy ${ }^{4} \cdot$ V. M. Job ${ }^{2}$ (D)
}

Received: 19 December 2019 / Accepted: 17 June 2020 / Published online: 25 June 2020

(c) Springer Nature Switzerland AG 2020

\begin{abstract}
The significance of aligned magnetic flux on free convective radiative nanofluid along a stretching surface with thermal energy storage is investigated in this paper. The problem has been modelled mathematically under Newtonian slip effect. The Prandtl boundary-layer expressions are framed using Lie group analysis and treated numerically. By employing the shooting method and the Runge-Kutta fourth-order technique, a solution to the dimensionless equations is obtained. The influence of pertinent dimensionless variables on the velocity, temperature, shear stress and heat transfer rate are scrutinized via graphical plots. The results of this study show that the velocity of the nanofluid is lower and wall shear flux is higher for greater aligned magnetic flux. More energy is transferred from the fluid system when multi-walled carbon nanotubes are immersed in the base liquid. The velocity of the nanofluid is decreased with increased slip parameter. Also, the temperature rises and heat flux is reduced with enhanced thermal energy storage/generation. The numerical solutions obtained in the present work are compared to existing literature, and they are found to be in a good agreement.
\end{abstract}

Keywords Aligned magnetic flux · Carbon nanotubes · Lie Group analysis · Navier slip · Radiation · Thermal energy storage

\section{List of symbols}

$B_{0} \quad$ Uniform magnetic flux density $(\mathrm{T})$

g Gravity

$N \quad$ Slip parameter

$f(\eta) \quad$ Dimensionless velocity

$k_{f} \quad$ Thermal conductivity

$\mathrm{k}^{*} \quad$ Coefficient of mean absorption

$\operatorname{Pr} \quad$ Prandtl number

$q_{w} \quad$ Wall heat flux

$q_{r} \quad$ Radiative heat flux

$R \quad$ Radiation parameter

$\operatorname{Re}_{x} \quad$ Local Reynolds number

$\bar{u} \quad$ Component of velocity parallel to $x$ direction

$(\mathrm{m} / \mathrm{s})$

$\bar{u}_{w} \quad$ Stretching velocity $(\mathrm{m} / \mathrm{s})$ $\bar{v}_{w} \quad$ Suction velocity $(\mathrm{m} / \mathrm{s})$

$\bar{v} \quad$ Component of velocity parallel to $y$ direction $(\mathrm{m} / \mathrm{s})$

$\bar{T} \quad$ Local temperature of the nanofluid $(\mathrm{K})$

$T \quad$ Dimensionless temperature

$T_{w} \quad$ Wall temperature

$T_{\infty} \quad$ Ambient temperature

$Q_{0} \quad$ Volumetric rate of heat generation

\section{Greek symbols}

$\alpha \quad$ Thermal diffusivity of the nanofluid $\left(\mathrm{m}^{2} \mathrm{~s}^{-1}\right)$

$\sigma^{*} \quad$ Stefan-Boltzmann constant

$\sigma \quad$ Electrical conductivity $(\mathrm{S} / \mathrm{m})$

$\rho \quad$ Density of the nanofluid $\left(\mathrm{kg} / \mathrm{m}^{3}\right)$

$\mu \quad$ Dynamic viscosity of the nanofluid (Pa s)

$v \quad$ Kinematic viscosity of the nanofluid $\left(\mathrm{m}^{2} \mathrm{~s}^{-1}\right)$

$\varangle$ V. M. Job, Victor.Job@sta.uwi.edu| 'Department of Mathematics, Sri Venkateswara Engineering College for Women, Tirupati, India. ${ }^{2}$ Department of Mathematics and Statistics, The University of the West Indies, St. Augustine, Trinidad and Tobago. ${ }^{3}$ Department of Mathematics, SAS, VIT University, Vellore, India. ${ }^{4}$ Department of Mathematics, SV University, Tirupati, India. 
$\xi \quad$ Inclination angle of the magnetic field

$\theta \quad$ Dimensionless temperature

$\phi \quad$ Nanoparticle volume fraction

$\eta \quad$ Similarity variable

\section{Subscripts}

$f \quad$ Base fluid (water)

nf Nanofluid

CNT Carbon nanotubes

\section{Abbreviations}

MHD Magnetohydrodynamics

MWCNT Multi-walled CNTs (carbon nanotubes)

SWCNT Single-walled CNTs (carbon nanotubes)

\section{Introduction}

In order to achieve enhancement of thermal conductivity in heat transfer systems, the suspension of nanometersized particles (nanoparticles) in a suitable base liquid is a major advancement in the thermodynamics field. Studying these fluid suspensions, termed as nanofluids, has been a prominent subject of investigation in recent times due to their importance in many industrial and engineering applications. In particular, miniaturizing the system is playing a prominent role today; for this reason, nanotechnology is a popular and booming area of research. In the Fullerene family, the best allotrope is the carbon nanotube (CNT), which has a long and hollow chemical structure. The CNT wall comprises atom-dense carbon sheets, called graphene. Two types of CNTs are available; namely, multi-walled carbon nanotubes (MWCNTs) and single-walled carbon nanotubes (SWCNTs). Their thermophysical properties are reliant on the alignment of the graphene sheet inside the tube and this causes them to behave like a metal or a semiconductor; the investigations regarding this have started in early $90 \mathrm{~s}$. Waqar et al. [1] presented the analysis of MHD heat transfer on CNTwater nanofluid past a static or moving wedge. Haq et al. [2] examined MHD flows of CNTs along a stretching surface with water, ethylene glycol (EG) and engine oil as base fluids. Mahantesh et al. [3] investigated the CNTs immersed MHD nanofluids along with Marangonic effect. Later, he extended his work by incorporating varying heat source [4]. Khan et al. [5] explored the impact of heat transfer past a moving wedge. Reddy et al. [6] studied the MHD flow past a non-linear stretching sheet. Obula Reddy et al. [7] worked on the analysis of single wall and multi wall CNTs over a vertical cone. Ramzan et al. [8] analyzed the entropy generation past a vertical cone with radiation. Job et al. [9] investigated an unsteady MHD flow of natural convection water-based alumina or single wall carbon nanotubes within a wavy trapezoidal enclosed space in the presence of viscous and Joule heating. Job and Gunakala [10] studied the influence of SWCNT's and Au-water based nanoparticles in mixed convection boundary layer flow within a grooved channel.

The research problem of the stretching sheet is of great importance and is applicable to various concrete situations involving metallurgical processes and polymer extrusion phenomena. Applications of these phenomena include continuous stretching, annealing, rolling, tinning of copper wires, manufacturing of polymer sheets, cooling of long metallic plates in a cooling path, the boundary layer flow along a liquid film during condensation processes [11, 12]. Khan and Pop [13] conducted an investigation on the boundary layer flow along a stretching sheet. Musa et al. [14] discussed the chemical reaction and thermal radiation impact on time-dependent nanoflow with varying fluid properties. Akbar et al. [15] explored the slip impact on MHD nanoflow along an extended sheet. Irfan et al. [16] worked on exponentially stretching sheet with MHD and variable fluid properties. Waini et al. [17] studied on a porous extending/spread out sheet. Rida et al. [18] investigated the combined effects of heat source/sink on radiative MHD nanoliquid. Poornima et al. [19] examined the influence of MHD on mixed convection temperature-dependent viscosity on the flow of a cylindrically-shaped copper water based nanofluid over a moving vertical surface. The hydromagnetic convective flow of a nanofluid over an exponential stretching surface with viscous dissipation effects was examined by Sreenivasulu and Poornima [20].

Magnetohydrodynamic (MHD) flows and slip phenomena receive considerable attention from researchers because of their distinct and widespread contribution to industrial and manufacturing processes. In general, the noslip condition produces erroneous results and is no longer valid [21-28]; this is particularly true when studying the cooling of microelectromechanical systems (MEMS). Further, the role of radiation in heat transportation has wider involvement in distinct branches of thermal engineering and industrial processes like gas turbines, aero-space vehicles, aircraft propulsion processes, missiles, nuclear power (thermal) plants and satellites [29-35].

In moving fluids, the investigation of heat source/sink is essential for the understanding of chemically-reacting systems and in cases where dissociation of the fluid occurs; in these situations, the temperature gradients produced within the fluid are significant. Possible heat sources influence heat and mass transfer phenomena in semiconductor wafers, etc. A Lie-group analysis on stagnation point flow of nanofluid past a stretching porous sheet with radiation and heat generation/absorption was presented by Sreenivasulu and Bhaskar Reddy [36]. Sreenivasulu and Bhaskar Reddy [37] extended their study by including the effects of chemical 
reaction. Burgers nanofluid with heat source/sink along a stretching sheet was investigated by Khan and Khan [38]. Very recently heat source/sink effect on MHD flow along a vertical stretching sheet was presented by lbrahim et al. [39]. Konda et al. [40] investigated the effect of heat generation/ absorption and melting heat on MHD Williamson nanofluid past a porous sheet.

To the authors' comprehension, the majority of the literature on nanoliquids considered MHD flows with heat sources/sinks. However, this paper is concerned with the aligned magnetic field impact on Navier slip flow of nanofluid imbedded carbon nanotubes along a stretching surface with thermal energy storage/heat source. Moreover, the similarity variables are obtained through Lie group analysis, and the comparisons of flow and heat transfer performance between SWCNTs and MWCNTs had not previously been considered. This is currently a booming topic as it has substantial medical and industrial applications such as nanofluidic pumps and micro-fluidic machinery. Thus, in our present work, we numerically examine the behavior of aligned MHD flow of nanofluid with immersed CNTs under the influence of radiation and Navier slip effects. The impact of pertinent physical controlled variables on velocity, friction, temperature factor (Nusselt number) and the streamlines are portrayed and analysed numerically.

\section{Mathematical analysis}

We consider a steady two-dimensional boundary layer flow of nanofluid along a vertical stretching sheet. The flow of nanofluid with imbedded carbon nanotubes is considered to be incompressible and laminar. The presence of an aligned magnetic field and thermal radiation are assumed, and thermal energy storage and internal heat generation are discussed in this paper. The flow is taken along the $\bar{x}$-axis (upward direction) and the sheet is elongated. The $\bar{y}$-axis is taken as being perpendicular to the sheet and inclined magnetic field is applied as shown in the flow diagram Fig. 1. The fluid temperature along the stretching surface is a constant value $T_{w}$ whereas the ambient temperature of the fluid is $T_{\infty}\left(T_{w}>T_{\infty}\right)$.

Based on the description of the problem, the equations that govern the Prandtl boundary layer flow is given as follows:

$\frac{\partial \bar{u}}{\partial \bar{x}}+\frac{\partial \bar{v}}{\partial \bar{y}}=0$

$\bar{u} \frac{\partial \bar{u}}{\partial \bar{x}}+\bar{v} \frac{\partial \bar{u}}{\partial \bar{y}}=\frac{1}{\rho_{n f}}\left[\mu_{n f} \frac{\partial^{2} \bar{u}}{\partial \bar{y}^{2}}+(\rho \beta)_{n f} g\left(\bar{T}-T_{\infty}\right)-\sigma_{n f} B_{0}^{2} \bar{u} \sin ^{2} \xi\right]$

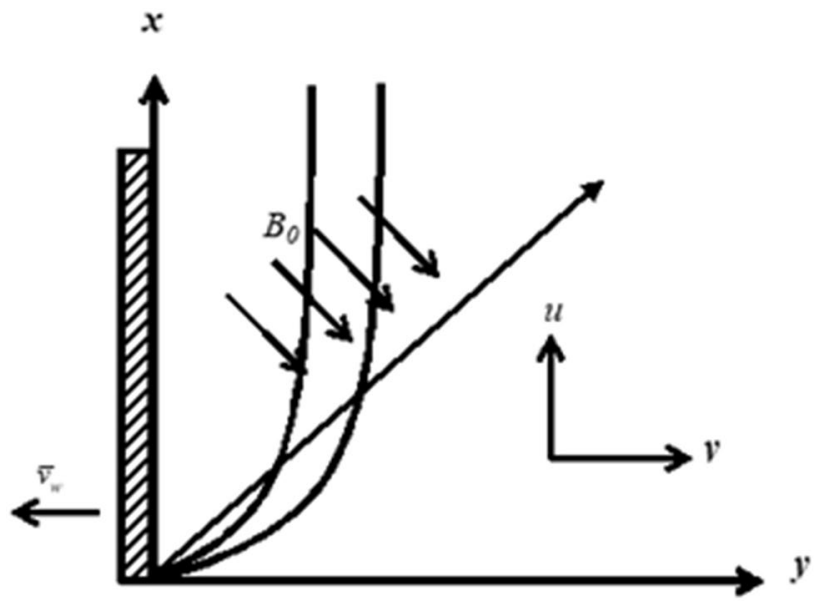

Fig. 1 Physical model and coordinate system

$\bar{u} \frac{\partial \bar{T}}{\partial \bar{x}}+\bar{v} \frac{\partial \bar{T}}{\partial \bar{y}}=\alpha_{n f} \frac{\partial^{2} \bar{T}}{\partial \bar{y}^{2}}-\frac{1}{\left(\rho C_{p}\right)_{n f}} \frac{\partial q_{r}}{\partial \bar{y}}+\frac{Q_{0}}{\left(\rho C_{p}\right)_{n f}}\left(\bar{T}-T_{\infty}\right)$

The corresponding conditions at the stretching surface and far from this surface are:

$\bar{u}=\bar{u}_{w}(\bar{x})+N \frac{\partial \bar{u}}{\partial \bar{y}}, \quad \bar{v}=\bar{v}_{w}, \quad \bar{T}=T_{w}$ at $\bar{y}=0$

$\bar{u} \rightarrow 0, \quad \bar{T} \rightarrow T_{\infty}$ as $\bar{y} \rightarrow \infty$

The radiative heat flux within the nanofluid is described (using the Rosseland approximation) by the following equation:

$q_{r}=-\frac{4}{3} \frac{\sigma^{*}}{k^{*}} \frac{\partial \bar{T}^{4}}{\partial \bar{y}}$

The Rosseland model is applicable to optically dense media, where radiation travels only a short distance before dispersed or immersed. When material has a great extinction coefficient, it can be treated as optically thick. We have taken a fluid model in which the energy transfer through radiation within optically dense nanofluid with CNTs exists before the heat is dispersed, so, radiative heat transfer is taken into account. By employing the Rosseland's approximation, the temperature variations inside the fluid are sufficiently small; thus Eq. (5) is linearized by extending $T^{4}$ into the Taylor's series expansion about $T_{\infty}$ which, upon ignoring higher-order terms yields the equation

$\bar{T}^{4} \cong 4 T_{\infty}^{3} \bar{T}-3 T_{\infty}^{4}$

Consequently, Eq. (3) becomes 
$\bar{u} \frac{\partial \bar{T}}{\partial \bar{x}}+\bar{v} \frac{\partial \bar{T}}{\partial \bar{y}}=\alpha_{n f}\left(1+\frac{4}{3} R\right) \frac{\partial^{2} \bar{T}}{\partial \bar{y}^{2}}+\frac{Q_{0}}{\left(\rho C_{p}\right)_{n f}}\left(\bar{T}-T_{\infty}\right)$

The thermophysical properties of CNT nanofluids are given by the following analytical models.

1. Viscosity

$\mu_{n f}=\frac{\mu_{f}}{(1-\phi)^{2.5}}$

\section{Density}

$\rho_{n f}=(1-\phi) \rho_{f}+\phi \rho_{C N T}$

\section{Heat capacity}

$\left(\rho C_{p}\right)_{n f}=(1-\phi)\left(\rho C_{p}\right)_{f}+\phi\left(\rho C_{p}\right)_{C N T}$

\section{Thermal expansion coefficient}

$(\rho \beta)_{n f}=(1-\phi)(\rho \beta)_{f}+\phi(\rho \beta)_{C N T}$

\section{Electrical conductivity}

$\sigma_{n f}=\sigma_{f}\left[1+\frac{3 \phi\left(\sigma_{s} / \sigma_{f}-1\right)}{\sigma_{s} / \sigma_{f}-2-\left(\sigma_{s} / \sigma_{f}-1\right) \phi}\right]$

\section{Thermal conductivity:}

At a small scale, proposed nanofluid models are applicable to circular or elliptic particles, which actually lag in exhibiting the spatial distribution properties. Xue [41] suggested the following model for CNTs, which are large axial ratio ellipsoids (Table 1):

$\kappa_{n f}=\kappa_{f} \frac{1-\phi+2 \phi \frac{\kappa_{C N T}}{\kappa_{C N T}-\kappa_{f}} \ln \frac{\kappa_{C N T}+\kappa_{f}}{2 \kappa_{f}}}{1-\phi+2 \phi \frac{\kappa_{f}}{\kappa_{C N T}-\kappa_{f}} \ln \frac{\kappa_{C N T}+\kappa_{f}}{2 \kappa_{f}}}$

We introduce the below similarity (dimensionless) variables:

Table 1 Thermo-physical properties of SWCNT, MWCNT and the base liquid

\begin{tabular}{llll}
\hline $\begin{array}{l}\text { Thermo physical } \\
\text { properties }\end{array}$ & $\begin{array}{l}\text { Base liquid } \\
\text { Water }\end{array}$ & \multicolumn{2}{l}{ Types of nanoparticles } \\
\cline { 3 - 4 } & SWCNT & MWCNT \\
\hline$\rho\left(\mathrm{kg} / \mathrm{m}^{3}\right)$ & 997 & 2600 & 1600 \\
$C_{p}(\mathrm{~J} / \mathrm{kg} \mathrm{K})$ & 4179 & 425 & 796 \\
$k(\mathrm{~W} / \mathrm{m} \mathrm{K})$ & 0.613 & 6600 & 3000 \\
$\beta \times 10^{-5}\left(\mathrm{~K}^{-1}\right)$ & 21 & 0.33 & 0.72 \\
$\sigma\left(\Omega^{-1} \mathrm{~m}^{-1}\right)$ & 0.05 & $4.8 \times 10^{7}$ & $3.3 \times 10^{6}$ \\
\hline
\end{tabular}

$x=\frac{\bar{x}}{L}, \quad y=G r^{1 / 4} \frac{\bar{y}}{L}, \quad u=G r^{-1 / 2} \frac{L \rho_{f}}{\mu_{f}} \bar{u}, \quad v=G r^{-1 / 4} \frac{L \rho_{f}}{\mu_{f}} \bar{v}, \quad T=\frac{\bar{T}-T_{\infty}}{T_{w}-T_{\infty}}$,

$\alpha_{n f}=\kappa_{n f} /\left(\rho C_{p}\right)_{n f}, \quad \bar{u}_{w}(\bar{x})=\frac{v_{f} G r^{1 / 2}}{L^{2}} \bar{x}, \quad R=16 \sigma * T_{\infty}^{3} / 3 k_{f} k *, \quad S=-\frac{\rho_{f} L \bar{v}_{w}}{\mu_{f} G r^{1 / 4}}$,

$G r=\rho_{f}^{2} g(\rho \beta)_{f}\left(T_{w}-T_{\infty}\right) L^{3} / \mu_{f}^{2}, \quad \operatorname{Pr}=\mu_{f} /\left(\rho_{f} \alpha_{f}\right), \quad M=\sigma_{f} B_{0}^{2} L^{2} /\left(\mu_{f} G r^{1 / 2}\right)$

$\gamma=\frac{G r^{1 / 4} N}{L}, \quad R e_{x}=\bar{x} \bar{u}_{w} / f$

Equations (1), (2) and (7) acquire the subsequent nondimensional form:

$\frac{\partial u}{\partial x}+\frac{\partial v}{\partial y}=0$

$u \frac{\partial u}{\partial x}+v \frac{\partial u}{\partial y}=\frac{\rho_{f}}{(1-\phi) \rho_{f}+\phi \rho_{C N T}}$

$\left\{\frac{1}{(1-\phi)^{2.5}} \frac{\partial^{2} u}{\partial y^{2}}+\operatorname{Gr}\left[1-\phi+\phi(\rho \beta)_{C N T} /(\rho \beta)_{f}\right] T-\sigma_{n f} / \sigma_{f} M u \sin ^{2} \xi\right\}$

$u \frac{\partial T}{\partial x}+v \frac{\partial T}{\partial y}$

$=\frac{4 R+3}{3 P r} \frac{\left(\rho C_{p}\right)_{f}}{(1-\phi)\left(\rho C_{p}\right)_{f}+\phi\left(\rho C_{p}\right)_{C N T}} \frac{\kappa_{n f}}{\kappa_{f}} \frac{\partial^{2} T}{\partial y^{2}}$

$+\frac{\lambda\left(\rho C_{p}\right)_{f}}{(1-\phi)\left(\rho C_{p}\right)_{f}+\phi\left(\rho C_{p}\right)_{C N T}} T$

The associated boundary conditions are:

$u=x+\gamma \frac{\partial u}{\partial y}, \quad v=-S, \quad T=1$ at $y=0$

$u \rightarrow 0, \quad T \rightarrow 0$ as $y \rightarrow \infty$

We define the stream function $\psi$ by

$u=\frac{\partial \psi}{\partial y}, \quad v=-\frac{\partial \psi}{\partial x}$

so that the equation of continuity is satisfied and the remaining equations take the form:

$\frac{\partial \psi}{\partial y} \frac{\partial^{2} \psi}{\partial x \partial y}-\frac{\partial \psi}{\partial x} \frac{\partial^{2} \psi}{\partial y^{2}}$

$$
\begin{aligned}
& =\frac{\rho_{f}}{(1-\phi) \rho_{f}+\phi \rho_{C N T}} \frac{1}{(1-\phi)^{2.5}} \frac{\partial^{3} \psi}{\partial y^{3}} \\
& +\frac{\rho_{f}}{(1-\phi) \rho_{f}+\phi \rho_{C N T}} \\
& \left\{G r\left[1-\phi+\phi(\rho \beta)_{C N T} /(\rho \beta)_{f}\right] T-\sigma_{n f} / \sigma_{f} M \frac{\partial \psi}{\partial y} \sin ^{2} \xi\right\}
\end{aligned}
$$




$$
\begin{aligned}
\frac{\partial \psi}{\partial y} \frac{\partial T}{\partial x}-\frac{\partial \psi}{\partial x} \frac{\partial T}{\partial y}= & \frac{\lambda\left(\rho C_{p}\right)_{f}}{(1-\phi)\left(\rho C_{p}\right)_{f}+\phi\left(\rho C_{p}\right)_{C N T}} T \\
& +\frac{4 R+3}{3 P r} \frac{\left(\rho C_{p}\right)_{f}}{(1-\phi)\left(\rho C_{p}\right)_{f}+\phi\left(\rho C_{p}\right)_{C N T}} \frac{\kappa_{n f}}{\kappa_{f}} \frac{\partial^{2} T}{\partial y^{2}}
\end{aligned}
$$

and the boundary conditions become

$$
\begin{aligned}
& \frac{\partial \psi}{\partial y}=x+\gamma \frac{\partial^{2} \psi}{\partial y^{2}}, \quad \frac{\partial \psi}{\partial x}=S, \quad T=1 \text { at } y=0 \\
& \frac{\partial \psi}{\partial y} \rightarrow 0, \quad T \rightarrow 0 \text { as } y \rightarrow \infty
\end{aligned}
$$

Equations (19) and (20) along with (21) are a highly nonlinear and coupled system of partial differential equations. To convert them into ordinary differential equations, we introduce the scaling Lie-group $\Gamma$ of transformations defined by $[42,43]$ :

$\Gamma: \quad x^{*}=x e^{\varepsilon \alpha_{1}}, \quad y^{*}=y e^{\varepsilon \alpha_{2}}, \quad \psi^{*}=\psi e^{\varepsilon \alpha_{3}}, \quad T^{*}=T e^{\varepsilon \alpha_{4}}$

Substituting Eq. (22) in Eqs. (19) and (20), we have

$$
\begin{aligned}
& e^{\varepsilon\left(\alpha_{1}+2 \alpha_{2}-2 \alpha_{3}\right)}\left(\frac{\partial \psi^{*}}{\partial y^{*}} \frac{\partial^{2} \psi^{*}}{\partial x^{*} \partial y^{*}}-\frac{\partial \psi^{*}}{\partial x^{*}} \frac{\partial^{2} \psi^{*}}{\partial y^{* 2}}\right) \frac{\rho_{f}}{(1-\phi) \rho_{f}+\phi \rho_{C N T}} \frac{1}{(1-\phi)^{2.5}} e^{\varepsilon\left(3 \alpha_{2}-\alpha_{3}\right)} \frac{\partial^{3} \psi^{*}}{\partial y^{* 3}} \\
&+\frac{\rho_{f}}{(1-\phi) \rho_{f}+\phi \rho_{C N T}}\left\{\operatorname{Gr}\left[1-\phi+\phi(\rho \beta)_{C N T} /(\rho \beta)\right]\right. \\
& e^{\varepsilon\left(\alpha_{1}+\alpha_{2}-\alpha_{3}-\alpha_{4}\right)}\left(\frac{\partial \psi^{*}}{\partial y^{*}} \frac{\partial T^{*}}{\partial x^{*}}-\frac{\partial \psi^{*}}{\partial x^{*}} \frac{\partial T^{*}}{\partial y^{*}}\right) \\
&=\frac{(R+3}{3 P r} \frac{\left(\rho C_{p}\right)_{f}}{(1-\phi)\left(\rho C_{p}\right)_{f}+\phi\left(\rho C_{p}\right)_{C N T}} e^{\varepsilon\left(2 \alpha_{2}-\alpha_{4}\right)} \frac{\kappa_{n f}}{\kappa_{f}} \frac{\partial^{2} T^{*}}{\partial y^{* 2}} \\
&+\frac{\lambda\left(\rho C_{p}\right)_{f}}{(1-\phi)\left(\rho C_{p}\right)_{f}+\phi\left(\rho C_{p}\right)_{C N T}} e^{-\varepsilon \alpha_{4} T^{*}}
\end{aligned}
$$$$
+\frac{\rho_{f}}{(1-\phi) \rho_{f}+\phi \rho_{C N T}}\left\{\operatorname{Gr}\left[1-\phi+\phi(\rho \beta)_{C N T} /(\rho \beta)_{f}\right] e^{-\varepsilon \alpha_{4}} T^{*}-e^{\varepsilon\left(\alpha_{2}-\alpha_{3}\right)} \sigma_{n f} / \sigma_{f} M \frac{\partial \psi^{*}}{\partial y^{*}} \sin ^{2} \xi\right\}
$$

Since Eqs. (23) and (24) are invariant under the Lie-group $\Gamma$, the relations between the parameters are as follows:

$\alpha_{1}+2 \alpha_{2}-2 \alpha_{3}=3 \alpha_{2}-\alpha_{3}=-\alpha_{4}=\alpha_{2}-\alpha_{3}$

$\alpha_{1}+\alpha_{2}-\alpha_{3}-\alpha_{4}=2 \alpha_{2}-\alpha_{4}=-\alpha_{4}$

From these relations, we get $\alpha_{3}=\alpha_{4}=\alpha_{1}$ and $\alpha_{2}=0$, which reduces $\Gamma$ to a single-parameter group of transformations

$x^{*}=x e^{\varepsilon \alpha_{1}}, \quad y^{*}=y, \quad \psi^{*}=\psi e^{\varepsilon \alpha_{1}}, \quad T^{*}=T e^{\varepsilon \alpha_{1}}$

By expanding the function $e^{\varepsilon \alpha_{1}}$ in powers of $\varepsilon$ using Taylor series and neglecting terms of order $\varepsilon$ or higher, Eq. (26) becomes

$x^{*}-x=x \alpha_{1} \varepsilon, \quad y^{*}-y=0, \quad \psi^{*}-\psi=\psi \alpha_{1} \varepsilon, \quad T^{*}-T=T \alpha_{1} \varepsilon$

$f^{\prime}(0)=1+\gamma f^{\prime \prime}(0), \quad f(0)=S, \quad \theta(0)=1$

$f^{\prime} \rightarrow 0, \quad \theta \rightarrow 0$ as $\eta \rightarrow \infty$

\section{Results and discussion}

The corresponding characteristic equations are

$\frac{d x}{x \alpha_{1}}=\frac{d y}{0}=\frac{d \psi}{\psi \alpha_{1}}=\frac{d T}{T \alpha_{1}}$

from which we obtain the similarity transformations

$\eta=y^{*}, \quad \psi^{*}=x^{*} f(\eta), \quad T^{*}=x^{*} \theta(\eta)$.

In view of Eq. (29), Eqs. (23) and (24) reduce to the ordinary differential equations

$f^{\prime \prime \prime}+(1-\phi)^{2.5}\left\{\begin{array}{c}{\left[1-\phi+\phi\left(\rho_{C N T} / \rho_{f}\right)\right]\left[f^{\prime \prime}-\left(f^{\prime}\right)^{2}\right]} \\ +G r\left[1-\phi+\phi(\rho \beta)_{C N T} /(\rho \beta)_{f}\right] \theta-\sigma_{n f} / \sigma_{f} M f^{\prime} \sin ^{2} \xi\end{array}\right\}=0$

$\frac{4 R+3}{3 P r} \frac{\kappa_{n f}}{\kappa_{f}} \theta^{\prime \prime}+\lambda \theta+\frac{(1-\phi)\left(\rho C_{p}\right)_{f}+\phi\left(\rho C_{p}\right)_{C N T}}{\left(\rho C_{p}\right)_{f}}\left[f \theta^{\prime}-f^{\prime} \theta\right]=0$

and the corresponding boundary conditions are:

It is necessary to study the engineering quantities such as coefficient of local skin friction $C_{f}$ and the local Nusselt number $\mathrm{Nu}_{x}$, which are defined as follows:

$C_{f}=\frac{\mu_{n f}}{\rho_{f} u_{w}^{2}}\left(\frac{\partial \bar{u}}{\partial \bar{y}}\right)_{\bar{y}=0}, \quad \mathrm{Nu}_{x}=\frac{\bar{x} \kappa_{n f}}{\kappa_{f}\left(T_{w}-T_{\infty}\right)}\left(-\frac{\partial \bar{T}}{\partial \bar{y}}\right)_{\bar{y}=0}$

Using Eqs. (14), (29) and (30), the shear stress and rate of heat transfer at the stretching sheet are described as

$\operatorname{Re}_{x}^{1 / 2} C_{f}=\frac{1}{(1-\phi)^{2.5}} f^{\prime \prime}(0), \quad \operatorname{Re}_{x}^{-1 / 2} N u_{x}=-\frac{\kappa_{n f}}{\kappa_{f}} \theta^{\prime}(0)$

The coupled non-linear ordinary differential Eqs. (30)-(31) and boundary conditions (32) were solved numerically by utilizing the fourth order R-K technique along with the 


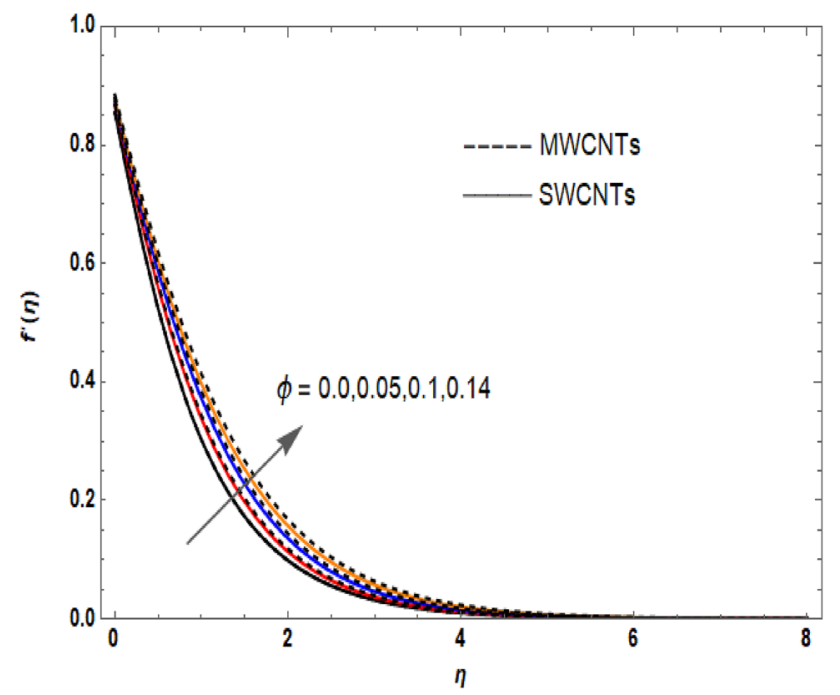

Fig. 2 Impact of $\phi$ on velocity

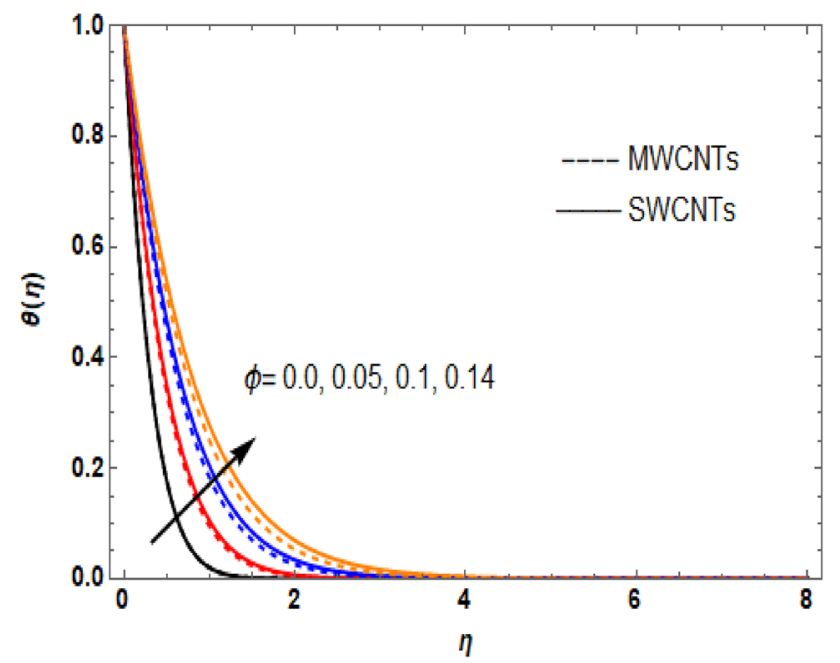

Fig. 3 Impact of $\phi$ on temperature

shooting method. The numerical algorithm was implemented using the Mathematica software. Firstly, the BVP concerning the physical model was transformed into an IVP consisting of five first-order differential equations and appropriate initial conditions. Applying the fourthorder R-K method, this IVP was solved numerically along with the shooting technique until the relative error is less than $10^{-6}$. Numerical results were obtained and analysed through graphs (Figs. 2, 3, 4, 5, 6, 7, 8, 9, 10, 11, 12, 13, 14, $15,16)$ and tables.

We validated the algorithm used in this study by making numerical comparisons with the works of Kalidas [44], Swathi Mukhopadyay [45] and Reddy et al. [46]. Numerical values of $f^{\prime \prime}(0)$ and $\theta^{\prime}(0)$ for varying Grashof number $\mathrm{Gr}$

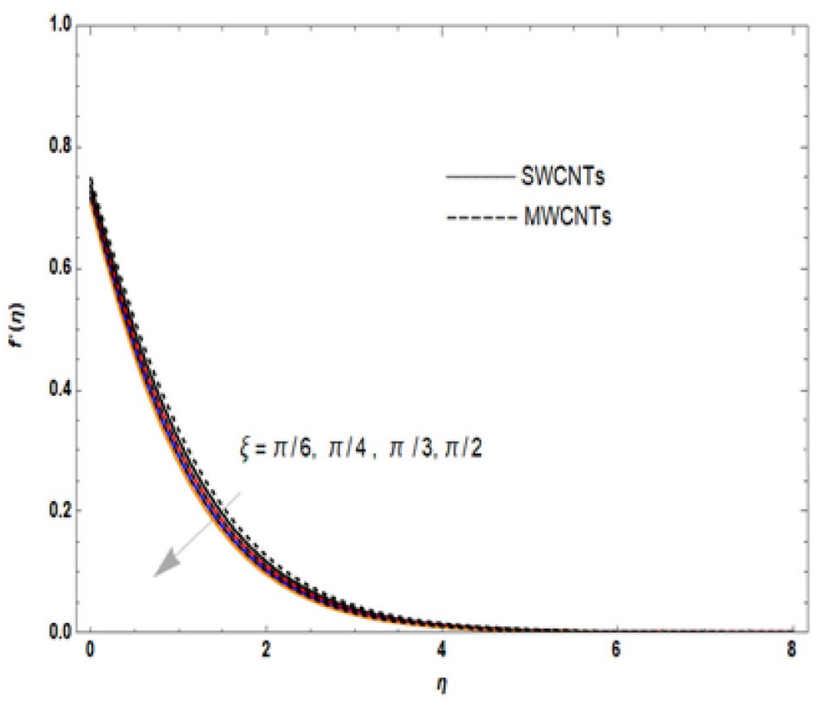

Fig. 4 Impact of $\xi$ on velocity

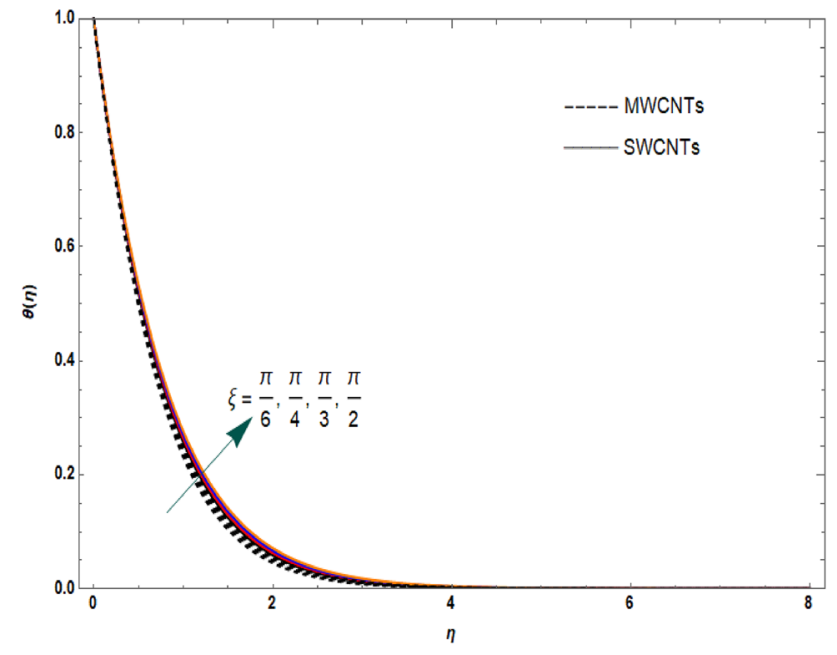

Fig. 5 Impact of $\xi$ on temperature

were displayed in Table 2, whereas Nusselt number values for different values of the Prandtl number Pr were shown in Table 3. It was found that the relative error in each of the compared values in Tables 2 and 3 is less than $0.1 \%$. Therefore, we determined that the results obtained using our present numerical algorithm have great consistency with the results of Kalidas [44], Swathi Mukhopadyay [45] and Reddy et al. [46].

In order to gain some physical insight into the present work, numerical results were computed with the following ranges of values: $0 \leq \phi \leq 0.2$ (for dilute nanoparticle suspensions); $0 \leq \xi \leq \frac{\pi}{2}$ (feasible range for the magnetic field inclination angle). The Prandtl number is chosen to be 6.785 , since the base fluid in the present work is water [44]. 


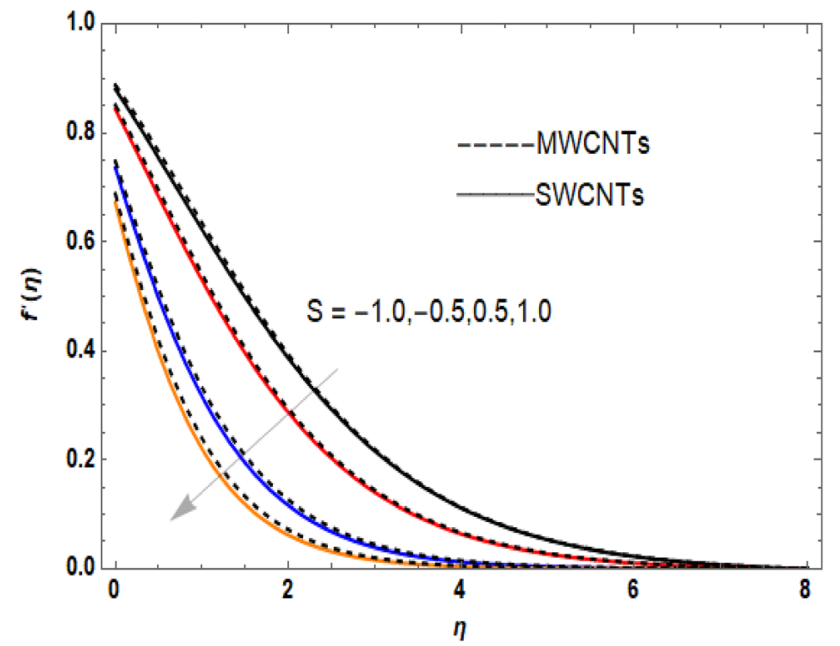

Fig. 6 Impact of $S$ on velocity

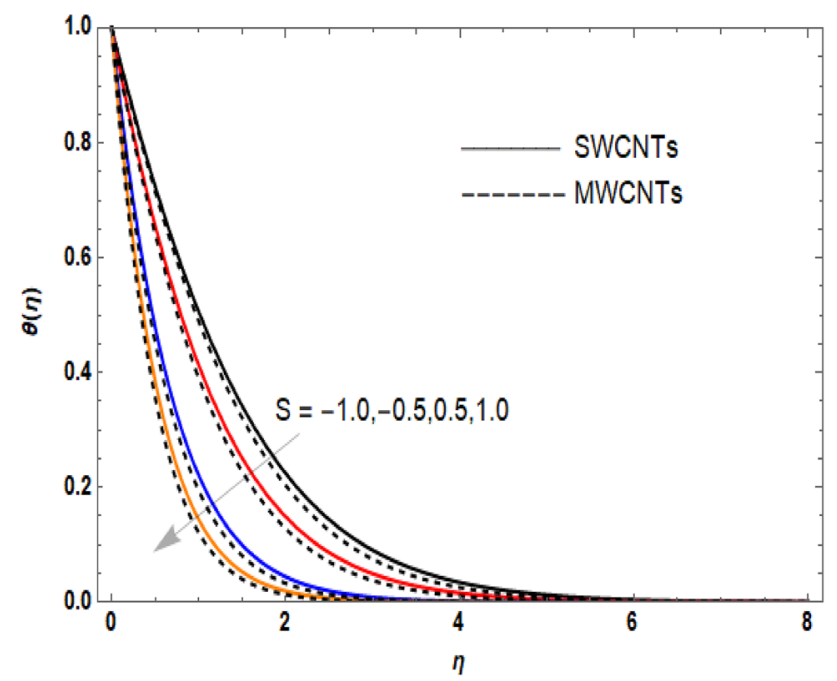

Fig. 7 Impact of S on Temperature

Figures 2 and 3 display the influence of nanotube volume fraction $\phi$ on the fluid velocity and temperature for SWCNTs and MWCNTs at $S=0.2, \lambda=0.1, \gamma=0.2, \mathrm{Gr}=1$, $\xi=\pi / 6$ and $M=0.5$. The velocity ascends (Fig. 2 ) for ascending $\phi$ values due to the enhancement of boundary layer thickness; this result is in agreement with Reddy et al. [46]. It is also seen that the nanofluid flow is faster in MWCNTs than SWCNTS. The nanoliquid temperature (Fig. 3) rises for rising $\phi$ due to the expansion in the thermal boundary layer thickness. This occurs since enhancing volume fraction of CNTs enhances the thermal conductivity and thus, the thickness of the thermal boundary layer also grows. Furthermore, heat transfer is greater for single-walled nanotubes in comparison to multi-wall nanotubes.

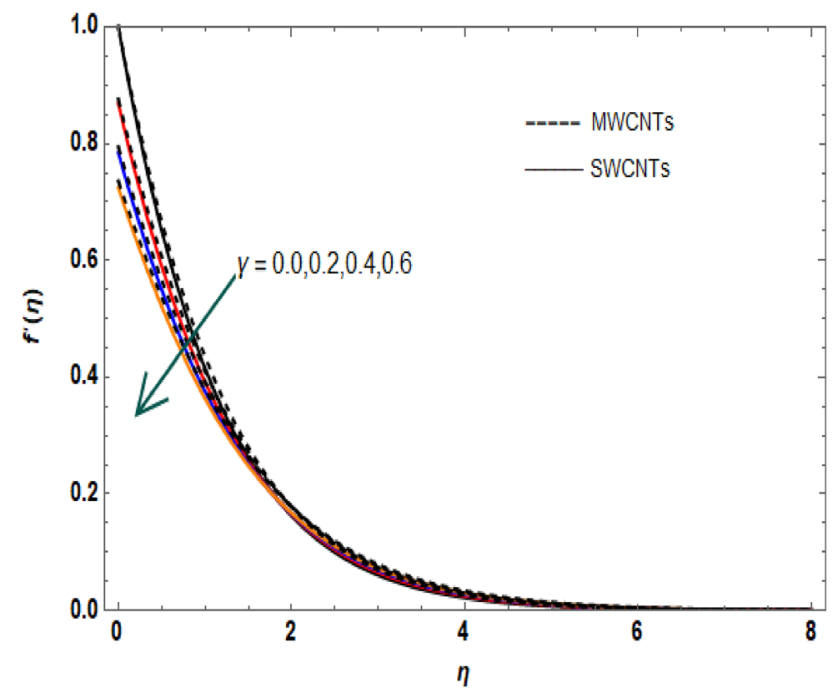

Fig. 8 Impact of $\gamma$ on velocity

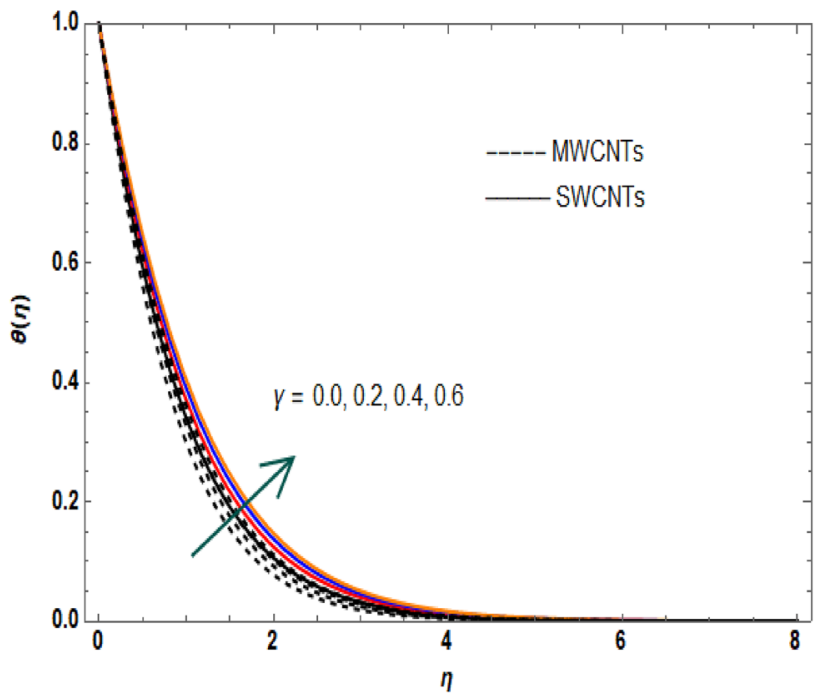

Fig. 9 Impact of $\gamma$ on Temperature

The aligned magnetic field inclination angle $\xi$ influence on the nanofluid velocity and temperature are portrayed in Figs. 4 and 5 when $S=0.2, \lambda=0.1, \gamma=0.2, \mathrm{Gr}=1, \phi=0.14$ and $M=0.5$. It is noticed in Fig. 4 that the fluid flow velocity is reduced with increasing magnetic field angle of inclination. This occurs since magnetic flux strengthens as aligned angle increases, which enhances the Lorentz force and supresses the fluid flow. Quite interestingly, it is seen that the temperature of the nanofluid (Fig. 5) is enhanced for an increase in angle of inclination. One can observe that multi-walled nanotubes are more dominant than single wall tubes in the case of velocity whereas the reverse trend is observed for temperature of the nanoliquid. 


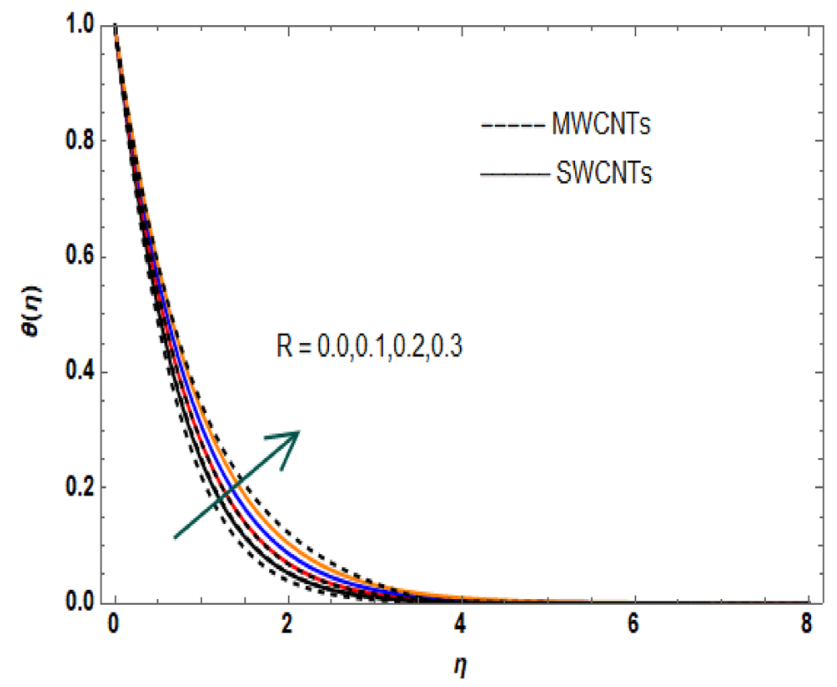

Fig. 10 Impact of $R$ Temperature

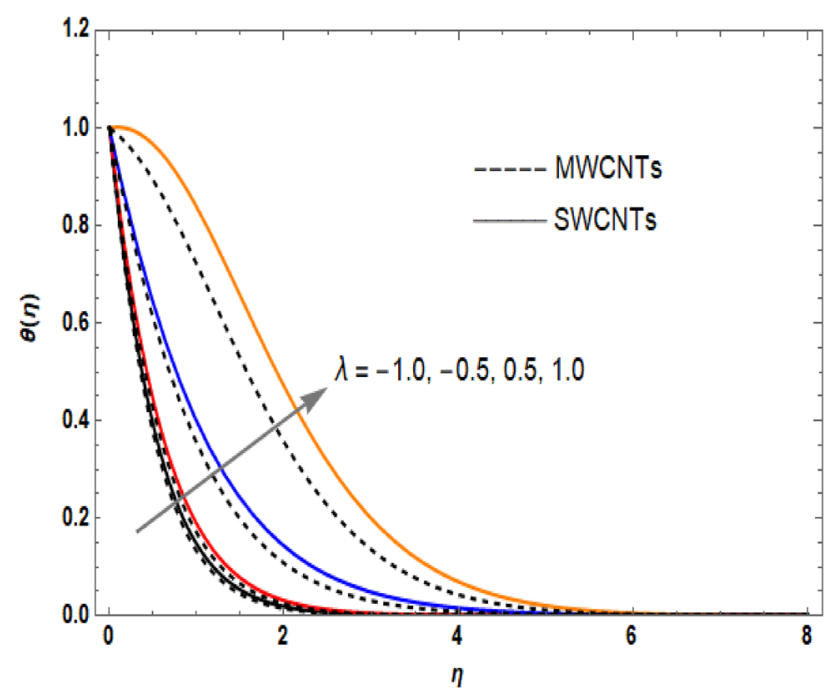

Fig. 11 Impact of $\lambda$ on Temperature

The effect of suction/injection parameter $S$ ( $S>0$ for suction and $\mathrm{S}<0$ for injection) on velocity and temperature profiles with $\lambda=0.1, \phi=0.14, \mathrm{Gr}=1, \xi=\pi / 6, \gamma=0.2$ and $M=0.5$ is depicted in Figs. 6 and 7. For both suction and injection, the velocity (Fig. 6) declines when S increases; this is consistent with the study conducted by Mukhopadhyay [45]. It is also noticed that MWCNTs plays a more dominant role than SWCNTs. In the same way, the temperature of the nanofluid (Fig. 7) decreases with increased values of $\mathrm{S}$. It is evident that the thickness of the thermal boundary layer reduces due to the movement of fluid towards the surface due to suction.

The influence of slip parameter $\gamma$ on velocity and temperature is displayed in Figs. 8 and 9 respectively.

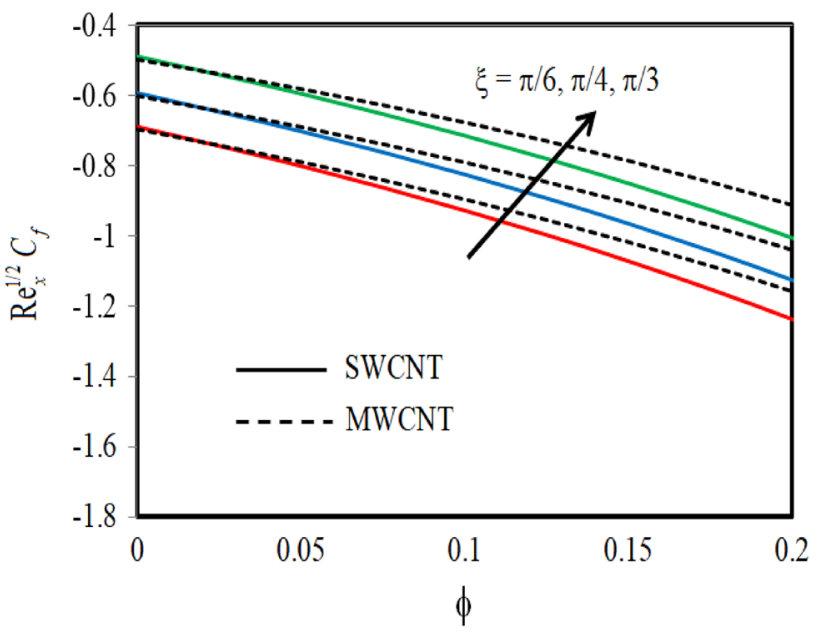

Fig. 12 Surface skin friction versus volume fraction for different values of $\xi$

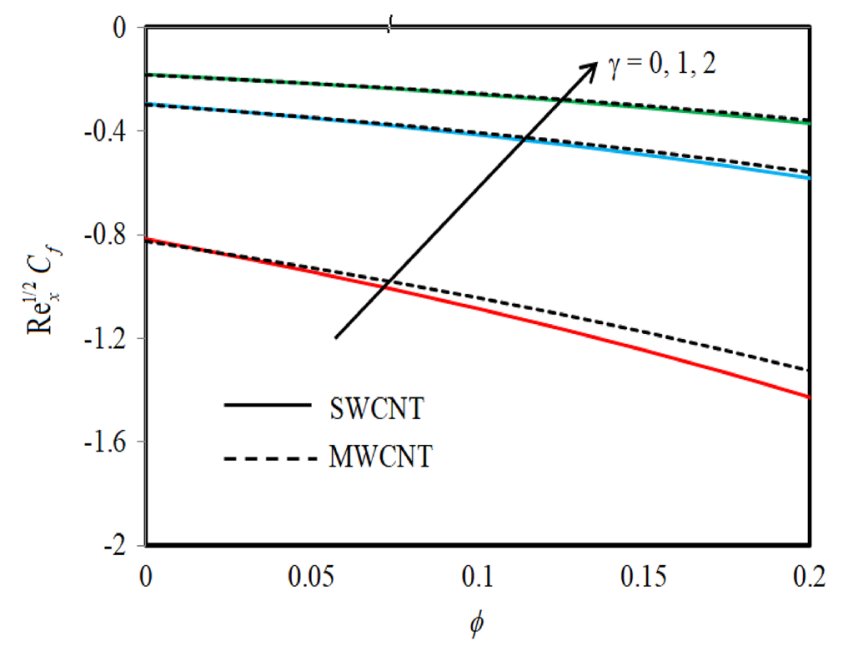

Fig. 13 Surface skin friction versus volume fraction for different values of $\gamma$

Escalating slip parameter escalates the slip at the stretching wall since a smaller amount of dispersion occurs inside the fluid, which thereby increases the momentum boundary layer thickness. Thus, increasing the slip parameter enhances the nanofluid velocity within the system. If $\gamma=0$, then no slip occurs, symbolizing that $f(0)=1$; i.e., the flow velocity is equal to the stretching surface velocity (Fig. 8). Furthermore, MWCNT exhibits a greater flow velocity in comparison with SWCNT. The fluid temperature (Fig. 9) rises with enhancing $\gamma$ due to increased thermal boundary layer thickness, and SWCNT has higher temperature values than MWCNT.

Figure 10 represents the impact of thermal radiation on temperature. The fluid temperature (Fig. 10) is enhanced with increased radiation parameter $R$. It is also evident that 


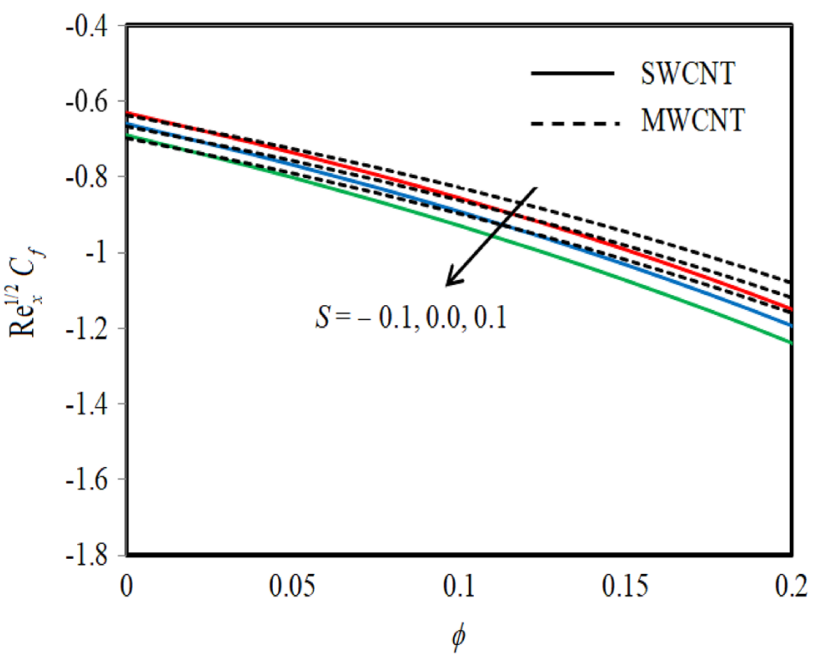

Fig. 14 Surface skin friction versus volume fraction for various $S$

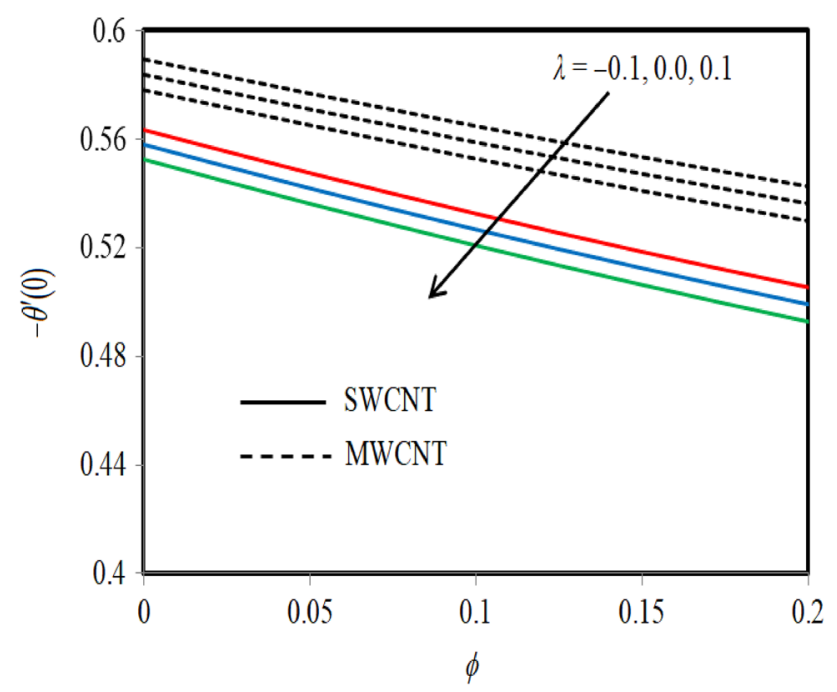

Fig. 15 Rate of heat transfer versus volume fraction for $\lambda$

single wall carbon nanotubes have the dominant character. Thermal variations for improving $\lambda(<0$ for generation and $>0$ for thermal energy storage) are depicted in Fig. 11 with parameter values $S=0.2, \gamma=0.2, \mathrm{Gr}=1, \xi=\pi / 6, \phi=0.14$ and $M=0.5$. In this figure, we noticed that the nanoliquid temperature rises as the thermal energy storage/heat source parameter $\lambda$ enhances. For $\lambda<0$ (source), the energy from the stretching sheet is absorbed by the fluid, which causes an increase in the thermal boundary layer thickness. Therefore, the temperature decreases and the heat energy is released into the fluid for $\lambda>0$ (sink), which also thickens the thermal boundary layer. Single-walled carbon nanotubes have a greater role in heat transfer from the fluid than multi-walled carbon nanotubes.

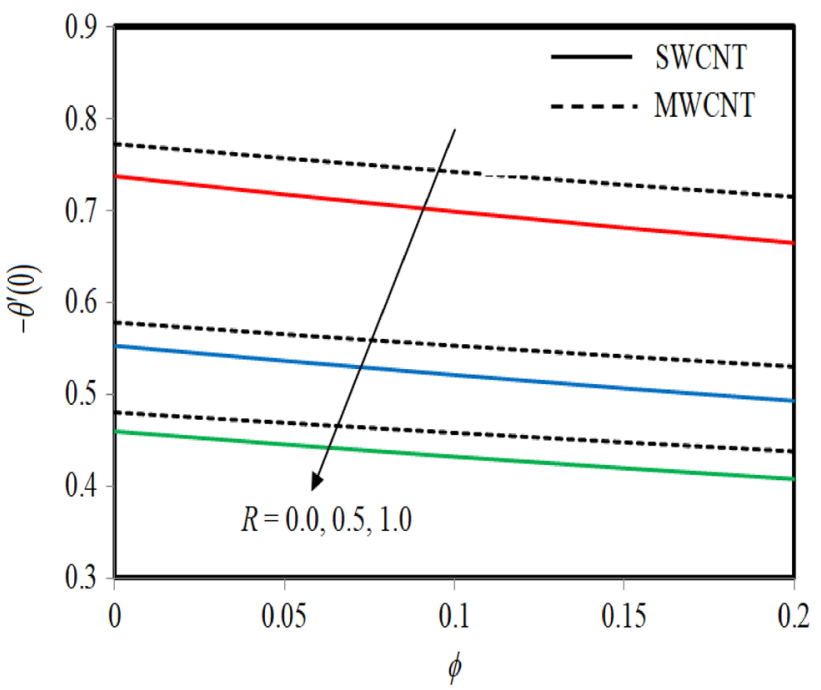

Fig. 16 Heat transfer rate versus volume fraction for varied $R$

The effects of various physical parameters on the surface skin friction at the stretching sheet are shown in Figs. 12,13 and 14. It is noticed in Fig. 12 that the skin friction decreases for larger nanotube volume fraction due to greater viscosity of the nanofluid; this result is in agreement with Kalidas [44]. We found that the skin friction is greater for increased aligned magnetic angle. Moreover, MWCNTs have higher skin friction values than SWCNTs. The same behaviour is observed for escalating slip parameter on the shear stress coefficient (Fig. 13); this is also consistent with the work of Kalidas [44]. From Fig. 14, it is seen that the rate of surface skin friction decays for growing volume fraction. And also it is evident that skin friction is reduced with increased suction/blowing.

Figure 15 reveals that the rate of heat transfer is higher for enhancing volume fraction, which is in agreement with the previous work of Reddy et al. [46]. This phenomenon is quite natural in nanofluid flow phenomena. From Fig. 15, it is clear that the heat transfer rate is decreased for rising thermal energy storage/source. Fascinatingly, SWCNT dominates the MWCNT in the case of heat transfer. The same behaviour is observed for increasing values of radiation parameter on rate of heat transfer, which is observed from Fig. 16.

\section{Conclusions}

This study focused on investigating the effects of aligned magnetic flux, thermal radiation and heat source/energy storage on a slip flow of water-carbon nanotube nanofluid along a vertical stretching sheet. A comparative study of multi-walled carbon nanotubes (MWCNTs) and 
Table 2 Comparison results for $f^{\prime \prime}(0)$ and $\theta^{\prime}(0)$ in some reduced cases

\begin{tabular}{llllllll}
\hline $\operatorname{Gr}$ & $f^{\prime \prime}(0)$ & & & $\theta^{\prime}(0)$ & & \\
\cline { 2 - 3 } & Present result & Kalidas [44] & Relative error (\%) & & Present result & Kalidas [44] & Relative error (\%) \\
\hline 0 & -1.0014211 & -1.001423 & $1.9 \times 10^{-4}$ & -2.3219232 & -2.321917 & $2.7 \times 10^{-4}$ \\
1 & -0.7629796 & -0.762983 & $4.5 \times 10^{-4}$ & & -2.3844349 & -2.384484 & $2.1 \times 10^{-4}$ \\
\hline
\end{tabular}

Table 3 Nusselt number for different Prandtl number in some reduced cases

\begin{tabular}{llllll}
\hline $\operatorname{Pr}$ & Present study & $\begin{array}{l}\text { Swathi Mukho- } \\
\text { padyay [45] }\end{array}$ & Relative error (\%) & Reddy et al. [46] & Relative error (\%) \\
\hline 1 & 0.954217 & 0.9547 & 0.0506 & 0.95485 & 0.0663 \\
2 & 1.471489 & 1.4714 & 0.0060 & 1.47144 & 0.0033 \\
3 & 1.869261 & 1.8691 & 0.0086 & 1.86906 & 0.0108 \\
5 & 2.500285 & 2.5001 & 0.0074 & 2.50012 & 0.0066 \\
10 & 3.660412 & 3.6603 & 0.0031 & 3.66037 & 0.0011 \\
\hline
\end{tabular}

single-walled carbon nanotubes (SWCNTs) was conducted and based on the computed numerical results, the following conclusions are drawn:

- The nanofluid flow velocity and skin friction along the stretching sheet are higher for MWCNTs than SWCNTs, but heat transfer is more effective with SWCNTs.

- The flow velocity is enhanced by increasing the CNT volume fraction and slip parameter, and reducing the Navier slip parameter, magnetic field inclination angle and suction/injection parameter.

- The temperature of the nanofluid is enhanced by increasing the magnetic field angle of inclination, thermal energy storage/source parameter and radiation parameter, whereas the temperature is reduced by increasing the suction/blowing parameter.

- The skin friction at the stretching sheet is higher with larger aligned magnetic angle, Navier slip parameter, and with smaller nanotube volume fraction and suction/blowing parameter.

- Heat transfer enhancement at the stretching sheet can be achieved by increasing the CNT volume fraction and decreasing the thermal energy storage/source parameter and radiation parameter.

The information contained in this work provides an improved understanding of the factors influencing slip flows of CNT-water nanofluids along a stretching sheet with convective heat transfer and an applied magnetic field. In particular, the results obtained herein regarding the magnetic field inclination angle and thermal energy storage/heat source for the cases of SWCNTs and MWCNTs are important contributions to the existing literature. For future work, we recommend that SWCNT and MWCNT nanofluids be modelled as non-Newtonian fluids with different types of non-linearly stretching sheets, as well as other geometrical configurations according to the physical nature of the industrial applications.

\section{Compliance with ethical standards}

Conflict of interest The authors declare that they have no conflict of interest.

\section{References}

1. Waqar A, Khan I, RichardCulham I, UIHaq R (2015) Heat transfer analysis of MHD water functionalized carbon nanotube flow over a static/moving wedge. J Nanomater. https://doi. org/10.1155/2015/934367

2. Haq RU, Khan ZH, Khan WA (2014) Thermophysical effects of carbon nanotubes on MHD flow over a stretching surface. Phys E Low-Dimens Syst Nanostruct 63:215-222

3. Mahanthesh B, Gireesha BJ, Shashikumar NS, Shehzad SA (2017) Marangoni convective MHD flow of SWCNT and MWCNT nanoliquids due to a disk with solar radiation and irregular heat source. Physica E 94:25-30

4. Mahanthesh B, Gireesha BJ, Animasaun IL, Muhammad T, Shashiku-mar NS (2019) MHD flow of SWCNT and MWCNT nanoliquids past a rotating stretchable disk with thermal and exponential space dependent heat source. Phys Scr 94(8):0031-8949

5. Khan WA, Culham R, Haq RU (2015) Heat transfer analysis of MHD water functionalized carbon nanotube flow over a static/ moving wedge. J Nanomater 2015, ID 934367

6. Reddy S, Reddy PBA, Chamkha AJ (2018) MHD flow analysis with water-based CNT nanofluid over a non-linear inclined stretching/shrinking sheet considering heat generation. Chem Eng Trans 71:1003-1008

7. Reddy YRO, Reddy MS, Reddy S (2018) MHD boundary layer flow of SWCNT-water and MWCNT-water nanofluid over a vertical cone with heat generation/absorption. Heat Transf Asian Res 48(8):539-555 
8. Ramzan M, Mohammad M, Howari F, Chung JD (2019) Entropy analysis of carbon nanotubes based nanofluid flow past a vertical cone with thermal radiation. Entropy 21:642

9. Job VM, Gunakala SR, Kumar BR, Sivaraj R (2017) Time-dependent hydromagnetic free convection nanofluid flows within a wavy trapezoidal enclosure. Appl Therm Eng 115:363-377

10. Job VM, Gunakala SR (2017) Mixed convection nanofluid flows through a grooved channel with internal heat generating solid cylinders in the presence of an applied magnetic field. Int J Heat Mass Transf 107:133-145

11. Nadeem S, Haq RU, Khan ZH (2014) Heat transfer analysis of water-based nanofluid over an exponentially stretching sheet. Alex Eng J 53:219-224

12. Zaimi Khairy, Ishak Anuar, Pop loan (2014) Boundary layer flow and heat transfer over a nonlinearly permeable stretching/ shrinking sheet in a nanofluid. Sci Rep 4:4404

13. Khan W, Pop I (2010) Boundary-layer flow of a nanofluid past a stretching sheet. Int J Heat Mass Transf 53(11-12):2477-2483

14. Mjankwi MA, Masanja VG, Mureithi EW, James MN (2019) Unsteady MHD flow of nanofluid with variable properties over a stretching sheet in the presence of thermal radiation and chemical reaction. Int J Math Math Sci ID 7392459

15. Akbar Tanvir, Batool Saima, Nawaz Rab, Zia Qazi Muhammad Zaigham (2019) Magnetohydrodynamics flow of nanofluid due to stretching/shrinking surface with slip effect. Adv Mech Eng 9(12):1-11

16. Irfan M, Farooq MA, Iqra $T$ (2019) Magnetohydrodynamic free stream and heat transfer of nanofluid flow over an exponentially radiating stretching sheet with variable fluid properties. Front Phys 7:186

17. Waini I, Ishak A, Pop I (2020) MHD flow and heat transfer of a hybrid nanofluid past a permeable stretching/shrinking wedge. Appl Math Mech Engl Ed 41:507-520

18. Rida SZ, Mohamed RA, Mubarak MS (2016) MHD nanofluid flow over a stretching sheet through a porous medium with heat generation and thermal radiation. J Nanofluids 5(1):130-138

19. Poornima T, Bhaskar Reddy N, Sreenivasulu P (2015) Temperature dependent viscosity effect on MHD mixed convective dissipating flow of cylinder shaped Cu-water nanofluid past a vertical moving surface. In: Proceedings of ICFM 2015, international conference on frontiers in mathematics 2015, March 26-28, 2015, Gauhati University, Guwahati, Assam, India

20. Sreenivasulu P, Poornima T (2015) Magneto hydrodynamic boundary layer flow of a dissipating nanofluid past an exponential stretching sheet with convective boundary condition. Math Sci Int Res J 4(2):288-292

21. Sheikholeslami M, Ellahi R, Vafai $\mathrm{K}$ (2018) Study of $\mathrm{Fe}_{3} \mathrm{O}_{4}$-water nanofluid with convective heat transfer in the presence of magnetic source. Alex Eng J 57:565-575

22. Souayeh B, Reddy MG, Sreenivasulu P, Poornima T, Rahimi-Gorji $M$ (2019) Comparative analysis on non-linear radiative heat transfer on MHD Casson nanofluid past a thin needle. J Mol Liq 284:163-174

23. Sheikholeslami M, Ellahi R, Shafee A, Li Z (2019) Numerical investigation for second law analysis of ferrofluid inside a porous semi annulus: an application of entropy generation and exergy loss. Int J Numer Methods Heat Fluid Flow 29(3):1079-1102

24. Poornima T, Sreenivasulu P, Reddy NB, Gunakala SR (2019) The effects of homo-/heterogeneous chemical reactions on Williamson MHD stagnation point slip flow: a numerical study, 157-165

25. Hassan M, Ellahi R, Bhatti MM, Zeeshan A (2019) A comparative study of magnetic and non-magnetic particles in nanofluid propagating over a wedge. Can J Phys 97(3):277-285

26. Sait RESM, Shehzad N, Mobin N (2019) Numerical simulation and mathematical modeling of electroosmotic Couette-Poiseuille flow of MHD power-law nanofluid with entropy generation. Symmetry 11(8):1038

27. Sreenivasulu P, Poornima T, Reddy NB, Reddy MG (2019) A numerical analysis on UCM dissipated nanofluid imbedded carbon nanotubes influenced by inclined Lorentzian force along with non-uniform heat source/sink. J Nanofluids 8(5):1076-1084

28. Ismail HNA, Abourabia AM, Hammad DA et al (2020) On the MHD flow and heat transfer of a micropolar fluid in a rectangular duct under the effects of the induced magnetic field and slip boundary conditions. SN Appl Sci 2:25

29. Sreenivasulu P, Poornima T, Bhaskar Reddy N (2018) Internal heat generation effect on radiation heat transfer MHD dissipating flow of a micropolar fluid with variable wall heat flux. J Nav Archit Mar Eng 15(1):53

30. Yousif MA, Ismael HF, Abbas T, Ellahi R (2019) Numerical study of momentum and heat transfer of MHD Carreau nanofluid over exponentially stretched plate with internal heat source/sink and radiation. Heat Transf Res 50(7):649-658

31. Alamri SZ, Ellahi R, Shehzad N, Zeeshan A (2019) Convective radiative plane Poiseuille flow of nanofluid through porous medium with slip: an application of Stefan blowing. J Mol Liq 273:292-304

32. Khan SA, Nie Y, Ali B (2020) Multiple slip effects on MHD unsteady viscoelastic nano-fluid flow over a permeable stretching sheet with radiation using the finite element method. SN Appl Sci 2:66

33. Gupta S, Kumar D, Singh J (2020) Analytical study for MHD flow of Williamson nanofluid with the effects of variable thickness, nonlinear thermal radiation and improved Fourier's and Fick's Laws. SN Appl Sci 2:438

34. Jha BK, Samaila G (2020) Thermal radiation effect on boundary layer over a flat plate having convective surface boundary condition. SN Appl Sci 2:381

35. Casson N (1959) A flow equation for pigment oil suspensions of the printing ink type. In: Mill CC (ed) Rheology of disperse systems. Pergamon Press, Oxford

36. Sreenivasulu P, Bhaskar Reddy N (2013) Radiation and mass transfer effects on boundary layer stagnation point flow of a nanofluid over a permeable stretching sheet in a porous medium with heat absorption/generation: a lie group analysis. Ann Math Phys 5:61-75

37. Sreenivasulu $P$, BhaskarReddy N (2013) Thermal radiation and chemical reaction effects on MHD stagnation-point flow of a nanofluid over a porous stretching sheet embedded in a porous medium with heat absorption/generation: Lie Group Analysis. J Glob Res Math Arch 1(7):13-27

38. Khan M, Khan WA (2016) Steady flow of Burgers nanofluid over a stretching surface with heat generation/absorption. J Braz Soc Mech Sci Eng 38:2359

39. Ibrahim MA, Ahmed GA, Osman M, Lund LA, Mossad BA, Hafedh $B$, Iskander T (2019) MHD flow and heat transfer over vertical stretching sheet with heat sink or source effect. Symmetry 11(3):297

40. Konda JR, Madhusudhana Reddy NP, Konijeti R, Dasore A (2019) Effect of non-uniform heat source/sink on MHD boundary layer flow and melting heat transfer of Williamson nanofluid in porous medium. Multidiscip Model Mater Struct 15(2):452-472

41. Xue QZ (2005) Model for thermal conductivity of carbon nanotube-based composites. Phys B 368:302-307

42. Tapanidis T, Tsagas G, Mazumdar HP (2003) Application of scaling group of transformations to visco-elastic second-grade fluid flow. Nonlinear Funct Anal Appl 8(3):345-350

43. Mukhopadhyay S, Layek GC, Samad SA (2005) Study of MHD boundary layer flow over a heated stretching sheet with variable viscosity. Int J Heat Mass Transf 48(21-22):4460-4466 
44. Kalidas D (2012) Slip flow and convective heat transfer of nanofluids over a permeable stretching surface. Comput Fluids 64:34-42

45. Mukhopadhyay Swati (2013) Slip effects on MHD boundary layer flow over an exponential stretching sheet with suction/blowing and thermal radiation. Ain Shams Eng J 4:485-491

46. BhaskarReddy N, Poornima T, Sreenivasulu P (2014) Influence of variable thermal conductivity on MHD boundary layer slip flow of ethylene-glycol based Cu nanofluids past a stretching sheet with convective boundary condition. Int J Eng Math 2014:905158

Publisher's Note Springer Nature remains neutral with regard to jurisdictional claims in published maps and institutional affiliations. 